Jules G. Becher

Gustaaf J. Lankhorst

Coen A. M. van Bennekom

Tanneke W. Vogelaar

\section{Measurement of the effect of a bolus dose of intrathecal baclofen by a repetitive movement test}

was obtained. The main outcome measures were RM test and clinical assessments of the Ashworth and spasm score. The results were (a) For the ankle a negative correlation was found between ROM and TSR of the flexor and extensors; for the knee a significant negative correlation was found only with the DSR of the biceps femoris. (b) A positive correlation was found between the Ashworth score and TSR of the extensors and between the spasm score and DSR and TSR of the gastrocnemius muscle. (c) Significant differences were found between baseline measurements and the optimal dose of baclofen for all measures. (d) A significant dose-dependent effect of intrathecal baclofen on the level of SRA was observed. The RM test is thus a useful clinical tool for objectively measuring the effect of intrathecal baclofen administration on spasticity in patients with an upper motor neuron syndrome.

Key words Intrathecal baclofen . Spasticity $\cdot$ Measurement
J. G. Becher ( $\square)$ · G. J. Lankhorst C. A. M. van Bennekom - T. W. Vogelaar Department of Rehabilitation Medicine, Vrije Universiteit, PO Box 7057, 1007 MB Amsterdam, The Netherlands e-mail: jg.becher@azvu.nl

Tel.: +31-20-4440760,

Fax: +31-20-4440787 with incremental doses $(25-150 \mu \mathrm{g})$ until an optimal effect or no effect

\section{Introduction}

Intrathecal baclofen is widely used to treat intractable spasticity in patients with upper motor neuron syndrome $[5,6$, 14, 16, 18-20, 24]. Clinicians use the term "spasticity" to refer to a wide spectrum of symptoms in upper motor neuron syndrome, such as abnormal reflexes and impaired movement. However, since 1980 the most widely accepted definition for spasticity has been "a motor disorder characterized by a velocity-dependent increase in tonic stretch reflexes with exaggerated tendon jerks resulting from hyperexcitability of the stretch reflex as one component of the upper motor neuron syndrome [13]. The term spasticity is used here in this context. Spasticity of the lower limbs can interfere with transfers, sitting, ambulation, rolling a 
wheelchair, driving a car, sexuality, self-care, personal hygiene activities, and sleeping and may cause pain $[1,6]$. Initial treatment includes physical therapy and oral administration of spasmolytic drugs, for example, baclofen, tizanidin, dantrolene sodium, and diazepam. When patients do not show a satisfactory response to this treatment, intrathecal baclofen administration with an implanted pump can be considered.

Prior to pump implantation, intrathecal bolus injections are given to rule out adverse reactions and to ensure the therapeutic effect of the drug on the spasticity and spasticity related problems. Trial screening on the effect of intrathecal baclofen with bolus administration is usually given with incremental doses [19] $(25-150 \mu \mathrm{g})$. This trial treatment is decisive for the implantation of a pump. The judgement of the effect of intrathecal baclofen administration on spasticity relies on subjective clinical assessments, such as the Ashworth score, spasm score, motor scores, assessment or observation of abilities, and recording of adverse effects [19].

Since the value of subjective assessment is limited, we feel it is of great importance to use an objective method of measurement to establish the effect of intrathecal baclofen administration. Only a combination of improvement in the clinical assessment of spasticity, functional improvements, and the objective measurement of spasticity provides an indication for the implantation a pump. In our Department we have developed a method to measure spasticity (restricted to the velocity-dependent resistance during passive movement). This is the repetitive movement (RM) test $[3,4]$. The objective of this study was to investigate various aspects of the validity of the passive RM test [25]. Four hypotheses were formulated:

1. Values on the RM test expressing the stretch reflex activity (SRA) show a negative relationship with the range of motion (ROM) in a joint. A decrease in spasticity is expected to be correlated with an increase in passive motion in a joint.

2. There are significant relationships between values on the RM test expressing spasticity and passive motion in a joint and the clinical assessment scales of tonus and spasticity.

3. RM results show a significant reduction in SRA after administration of the clinically optimal dose of baclofen in patients who show a positive response. Intrathecal administration of baclofen suppresses SRA.

4. RM results show a dose-dependent effect of intrathecal baclofen on spasticity. The level of spasticity depends on the patient characteristics and the dose of baclofen administered.

\section{Patients and methods}

Twenty-four patients ( 12 men, 12 women; mean age 41.8 years, range 18-54) were selected for trial treatment with intrathecal baclofen. All had suffered from a paraplegia or tetraplegia for at least 12 months, caused by an upper motor neuron lesion (multiple sclerosis $n=18$, spinal cord injury $n=4$, subarachnoidal bleeding $n=$ 1 , traumatic brain injury $n=1$ ). Patients were included if they had functional problems probably due to spasticity (defined as a velocity-dependent increase in resistance to passive movement) [13] Full written consent, according to the Declaration of Helsinki, was obtained from all the patients.

Patients were assessed by the Ashworth scale [2] and the spasm frequency scale $[6,18]$. Patients with multiple sclerosis were rated according to Kurtzke's Disability Status Scale [12], and those with spinal cord injury were evaluated by the American Spinal Injury Association Impairment Scale [7]. The functional problems due to spasticity, as reported by the patient or the care-givers, were also recorded. The most frequently reported problems before treatment concerned transfer from a wheelchair $(n=15)$, sitting $(n=14)$, washing and dressing $(n=13)$, sleeping $(n=6)$, and pain $(n=6)$. Four patients had an indoor walking capacity with crutches, 14 were restricted to wheelchair, and 6 were fully ADL dependent.

\section{Repetitive movement test}

The RM test consists of passive, alternating flexion and extension movements in the knee or ankle, imposed by an investigator keeping pace with a metronome at $69-100 \mathrm{bpm}(0.57-0.83 \mathrm{~Hz})$ for $30 \mathrm{~s}$. In the case of severe spasticity, the lowest frequency was chosen. ROM in a joint was recorded by a potentiometer-based goniometer. Muscle activity was recorded by surface electromyography
Fig. 1 Instrumentation for the measurement of the RM test. $A D C$ Analog-to-digital converter; $S R-E M G$ smoothed-rectified EMG

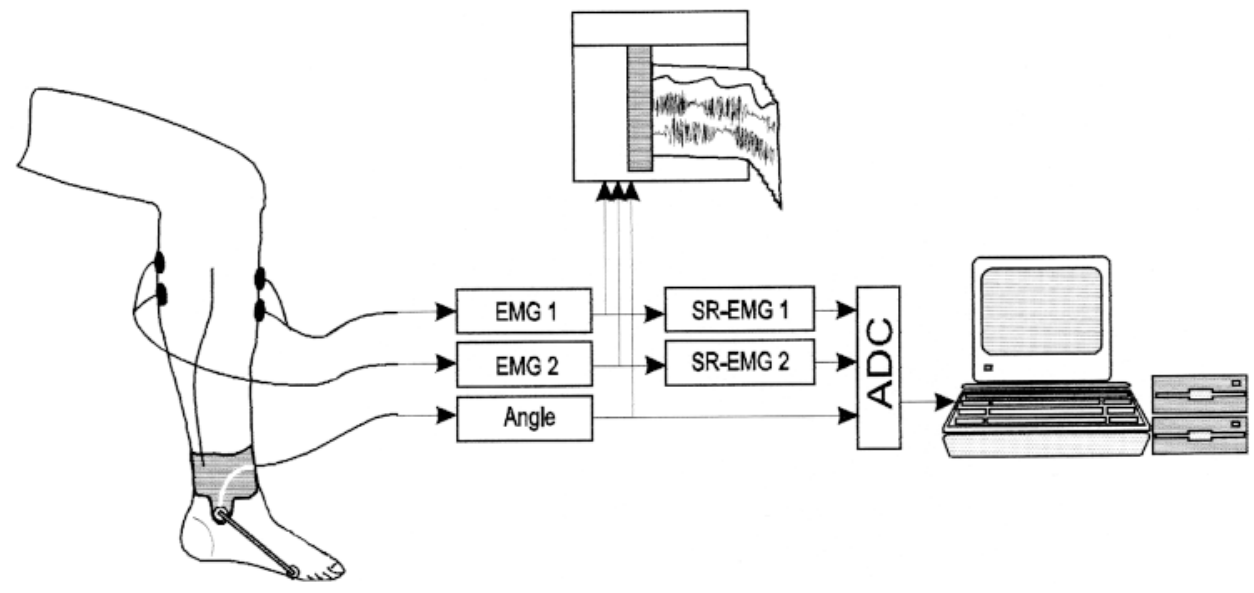


(EMG) of a flexor muscle (anterior tibial muscle or biceps femoris muscle), and one or two extensor muscles (soleus muscle and medial head of the gastrocnemius muscle or quadriceps femoris muscle; Fig. 1).

In healthy subjects EMG activity is virtually absent in passive movements [3]. The frequencies used in this test are too low to activate SRA $[9,17]$. For this reason the presence of EMG activity in a muscle can be considered as a symptom of raised SRA, a sign of spasticity. The enhanced stretch reflex during imposed movements can be present either in a dynamic way, i.e., only during passive stretching of a muscle (dynamic stretch reflex activity, DSR), or in a tonic way (tonic stretch reflex activity, TSR), i.e., showing EMG activity as long as passive movement is imposed, both in flexion and extension movements [22]. The DSR causes clinical symptoms, such as the "clasp knife" phenomenon and clonus. The TSR results in the clinical observation of stiffness during passive movement in a joint.

The RM test was performed in the clinically most spastic muscles of the lower extremities. When the biceps femoris or quadriceps muscles were the most spastic, the passive RM test of the knee was performed; when the triceps surae or anterior tibial muscle were the most spastic, the RM test of the ankle was performed. Two joints were always measured. Repeated measurements of a patient took place with the same frequency of movement. All patients were measured by the same investigator. The test lasted for approximately $20 \mathrm{~min}$. Depending on the quality of sitting balance, the test was performed with the patient sitting in the wheelchair or lying in bed, but always in the same position.

After positioning a spinal catheter, baseline measurements were performed. On all subsequent days at 8.00 a.m., $5 \mathrm{ml}$ fluid was administered intrathecally, and at 10.00 a.m. the patient was measured. The patient, nursing staff, physiotherapist, and laboratory staff were blinded for the treatment. All reported on the spasticity and the perceived problems related to spasticity. Baclofen was administered with increasing doses $(25-150 \mu \mathrm{g})$. The treatment was terminated when the effect of intrathecal baclofen on the spasticity and the functional problems was obvious.

\section{Data-processing}

For the bipolar lead-off of the surface EMG the electrodes were placed in the direction of the muscle fibers at the most central part of the palpable muscle belly. Rim-to-rim distance of the pair of electrodes was $23 \mathrm{~mm}$, the effective lead-off surface of the Meditrace Pellet electrodes was $1 \mathrm{~cm}^{2}$. The EMG was preamplified, high-pass filtered, amplified, and rectified to obtain the smoothed- rectified EMG (SR-EMG, equivalent to the integrated EMG). The SR-EMG of the muscles, together with the goniometer signal were stored online on a microcomputer using A/D conversion (sample frequency $120 \mathrm{~Hz}$ ).

Off-line, the movement was divided into cycles in order to calculate the movement frequency. The cycles were ensemble averaged for all signals to obtain the average movement and muscle activation patterns, together with the standard deviation, as a function of the cycle phase (0-100\%; Fig. 2) [23].

In the plots, when the maximum value of the SR-EMG exceeded $40 \mu \mathrm{V}$, it was normalized to a percentage of the recorded maximum SR-EMG value of each muscle. To quantify the DSR of a muscle the average maximal EMG activity of a muscle during passive stretch (in microvolts) was used, and to quantify the TSR the average minimal EMG activity of a muscle (in microvolts) was used, expressing the ability to relax a muscle during passive movement. The ROM was calculated from the goniometer signal as the mean difference between maximal flexion and maximal extension in a joint (Fig. 3).

\section{Statistics}

The statistics used were Spearman's rank correlation coefficient between the clinical assessments and RM results, the paired $t$ test to calculate the difference between baseline measurements of the RM test and the optimal dose of baclofen, and general linear model analysis of variance to compare of the various doses on SRA. Data were analyzed using the SPSS-PC statistical package, version 7.5 .

\section{Results}

The results are presented below in relation to the formulated hypotheses:

1. "Values on the RM test expressing the SRA show a negative relationship with the ROM in a joint." In the knee a significant correlation was found between the DSR biceps femoris and the ROM (Table 1). The TSR activity of the anterior tibial muscle, soleus muscle, and gastrocnemius muscle all showed a significant, negative correlation with the ROM in the ankle (Table 2).
Fig. 2 RM test: off-line signal processing. $S R-E M G$ Smoothed rectified EMG segmentation
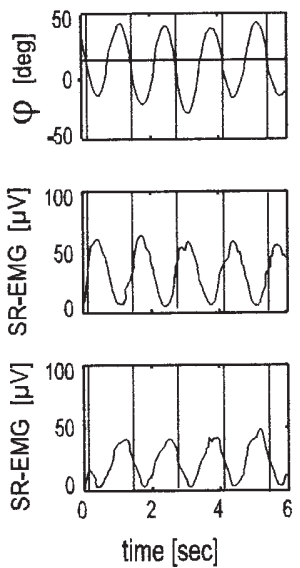

normalisation
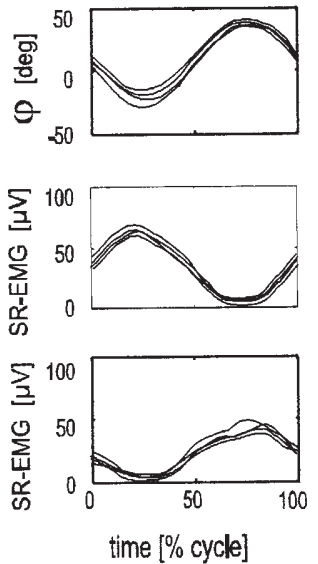

ensemble-averaging

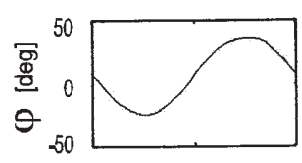

ankle

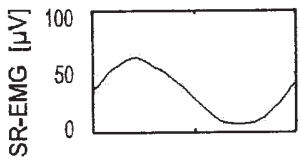

m. tibialis

anterior

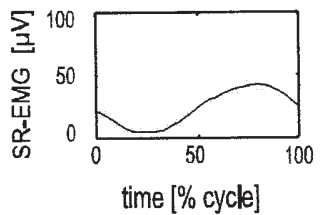

m. triceps

surae 
Fig. 3 Graph of a passive RM test of the ankle. TAM, Anterior tibial muscle; $S M$ soleus muscle; $G M$ gastrocnemius muscle; $F R Q$ frequency; $R O M$ range of motion; $S R-E M G$ smoothed-rectified EMG; MAX maximal EMG activity, dynamic stretch reflex activity; MIN minimal EMG activity, tonic stretch reflex activity. Ankle position: $90^{\circ}=0^{\circ}$ dorsiflexion. Clonus activity of the GM and SM during stretch is present. The TAM shows simultaneous EMG activity

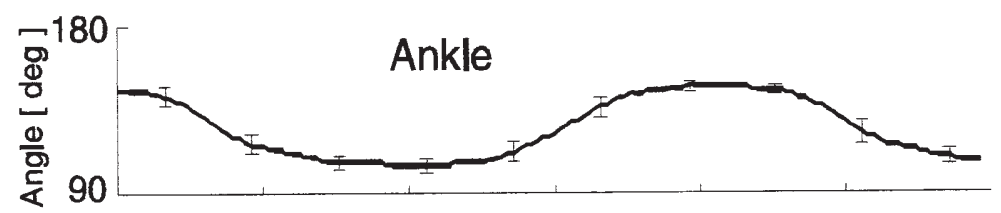

ROM: $44+/-3$ deg.

FRQ: $0.85+/=0.13 \mathrm{~Hz}$

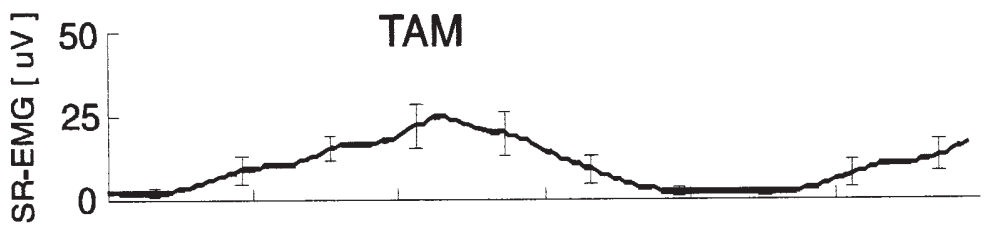

MAX: $24.8 \mathrm{uV}$

MIN: $2.0 \mathrm{uV}$

\section{SM}

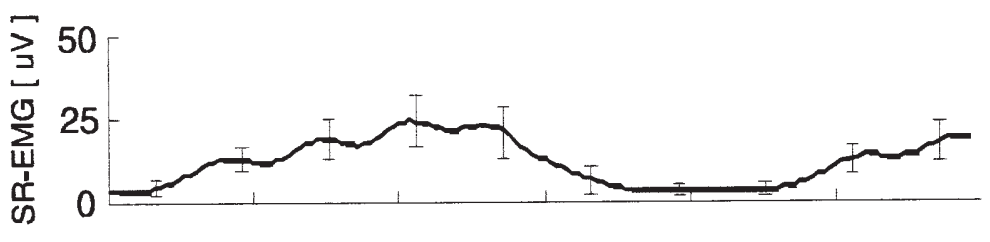

GM

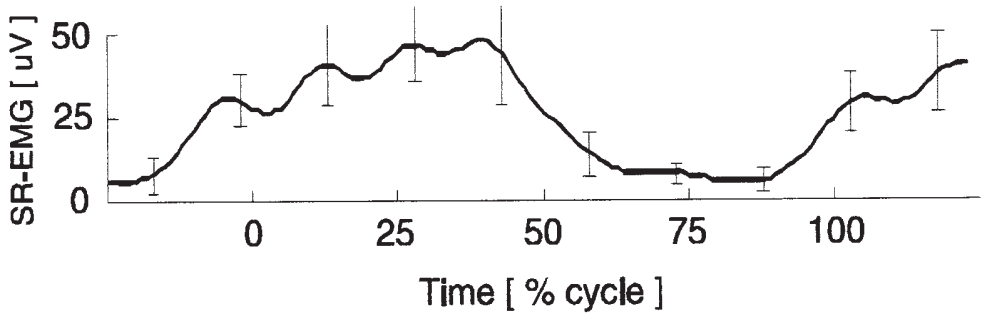

MAX: $48.2 \mathrm{uV}$

MIN: $\quad 5.5 \mathrm{uV}$
Table 1 Effect of baclofen on SRA and the correlation between ROM and SRA in the RM test: knee

\begin{tabular}{lccccc}
\hline & \multicolumn{2}{c}{ Biceps femoris } & & \multicolumn{2}{c}{ Quadriceps } \\
\cline { 2 - 3 } \cline { 6 - 7 } & DSR & TSR & & DSR & TSR \\
\hline Baclofen 0 $\mu \mathrm{g}(n=21)$ & $26 \pm 32$ & $15 \pm 19$ & & $42 \pm 50$ & $14 \pm 18$ \\
Baclofen 25 $\mu \mathrm{g}(n=16)$ & $18 \pm 15$ & $7 \pm 6$ & & $26 \pm 32$ & $8 \pm 12$ \\
Baclofen 50 $\mu \mathrm{g}(n=13)$ & $7 \pm 11$ & $3 \pm 4$ & $17 \pm 19$ & $5 \pm 6$ \\
Baclofen $>50 \mu \mathrm{g}(n=4)$ & $17 \pm 19$ & $3 \pm 5$ & & $9 \pm 4$ & $2 \pm 1$ \\
SRA-ROM, $\rho(n=21)$ & -0.623 & -0.235 & & -0.193 & -0.337 \\
$P$ & $0.003 *$ & 0.319 & & 0.403 & 0.135 \\
\hline
\end{tabular}

2. "There are significant relationships between values on the RM test expressing spasticity and passive motion in a joint and the clinical assessment scales of tonus and spasticity." The Ashworth tonus score had a weak but significant correlation with the TSR of the extensor (Spearman's $\rho=293, P=0.035$ ); a high Ashworth score was correlated with high TSR. The clinical spasm score was significantly correlated only with the DSR and the TSR of the gastrocnemius muscle (DSR, Spearman's $\rho=363, P=0.038$; TSR, $\rho=408, P=0.018$ ). Correlations between the clinical spasm score and the level of stretch activity of the quadriceps muscle, the biceps femoris muscle, the soleus muscle, and the anterior tibial muscle were positive, as expected, but not statistically significant.

3. "RM results show a significant reduction in SRA after administration of the clinically optimal dose of baclofen in patients who show a positive response." All scores expressing TSA and DSR decreased significantly with the clinically optimal dose of baclofen, both in the extensors and flexors of the knee and ankle (Table 3).

4. "RM results show a dose-dependent effect of intrathecal baclofen on spasticity." A significant effect of the dose of intrathecal baclofen was demonstrated (Tables 1 and 2). All scores expressing spasticity decreased significantly with an increasing dose baclofen (results of analysis of variance, $P<0.014$, data not shown). 
Table 2 Effect of baclofen on SRA and the correlation between ROM and SRA in the RM test: ankle

$* P<0.05$

\begin{tabular}{|c|c|c|c|c|c|c|}
\hline & \multicolumn{2}{|c|}{ Tibial anterior } & \multicolumn{2}{|l|}{ Soleus } & \multicolumn{2}{|c|}{ Gastrocnemius } \\
\hline & DSR & TSR & DSR & TSR & DSR & TSR \\
\hline Baclofen $0 \mu \mathrm{g}(n=44)$ & $34 \pm 26$ & $12 \pm 16$ & $16 \pm 16$ & $8 \pm 8$ & $16 \pm 14$ & $9 \pm 6$ \\
\hline Baclofen $25 \mu \mathrm{g}(n=38)$ & $15 \pm 19$ & $3 \pm 5$ & $4 \pm 4$ & $3 \pm 2$ & $5 \pm 5$ & $3 \pm 2$ \\
\hline Baclofen $50 \mu \mathrm{g}(n=19)$ & $11 \pm 12$ & $5 \pm 10$ & $6 \pm 6$ & $4 \pm 6$ & $8 \pm 7$ & $6 \pm 8$ \\
\hline Baclofen $>50 \mu \mathrm{g}(n=5)$ & $30 \pm 22$ & $16 \pm 17$ & $10 \pm 10$ & $10 \pm 11$ & $13 \pm 8$ & $13 \pm 9$ \\
\hline SRA-ROM, $\rho(n=44)$ & -0.226 & -0.342 & -0.236 & -0.378 & -0.205 & -0.397 \\
\hline$P$ & 0.145 & $0.025^{*}$ & 0.123 & $0.012 *$ & 0.236 & $0.018 *$ \\
\hline
\end{tabular}

Table 3 Change in spasticity between baseline measurement and optimal dose of baclofen (paired $t$ test)

\begin{tabular}{lccc}
\hline Parameter & $\begin{array}{l}\text { Mean } \pm \mathrm{SD} \\
(\mu \mathrm{V})\end{array}$ & $P$ & $\begin{array}{c}\text { 95\% CI of the } \\
\text { difference }\end{array}$ \\
\hline $\begin{array}{l}\text { Extensor } \\
\quad \text { DSR }\end{array}$ & & 0.002 & $6.8-28.9$ \\
$\quad$ Baseline & $26.1 \pm 38.4$ & & \\
Optimum & $8.3 \pm 13.0$ & & \\
TSR & & 0.000 & $4.3-12.3$ \\
Baseline & $11.7 \pm 13.7$ & & \\
Optimum & $3.4 \pm 5.0$ & & \\
Gastrocnemius & & & \\
DSR & & & \\
Baseline & $15.5 \pm 14.6$ & & \\
Optimum & $7.0 \pm 6.8$ & & \\
TSR & & 0.000 & \\
Baseline & $8.5 \pm 6.2$ & & \\
Optimum & $3.7 \pm 5.0$ & & \\
Flexor & & & \\
DSR & & & \\
Baseline & $34.6 \pm 26.3$ & & \\
Optimum & $13.1 \pm 18.9$ & & \\
TSR & & & \\
Baseline & $13.7 \pm 18.6$ & & \\
Optimum & $3.2 \pm 6.2$ & & \\
\hline
\end{tabular}

\section{Discussion}

The findings of this study confirm the relationship between scores expressing spasticity and the ROM in the ankle; the ROM in the ankle negatively correlated with TSR. DSR showed a significant negative correlation only in the biceps femoris muscle. A raised DSR was found only in some of the patients, as reflected by the large standard deviations in Table 1. Obviously the dynamic component of the increased SRA plays a minor role in limiting joint mobility in passive movements. This indicates that the limitation of the ROM is more likely caused by structural changes (muscle shortening and knee flexion contractures) than by spasticity since the levels of EMG activity decreased with an increasing dose of baclofen (Table 1).

A few significant correlations are found between the clinical scores of tonus and spasticity and the scores cal- culated from the RM test. In upper motor neuron syndromes not only the presence of spasticity contributes to the increased stiffness of a muscle but also biomechanical changes, such as stiffness from increased friction and stiffness caused by muscle shortening $[10,15]$. The presence of a thixotrophic substance (a substance with a nonNewtonian behavior, which displays reduced viscosity with an increased rate of shear deformation) has been postulated [21]. The lack of correlation between the Ashworth tonus score and ROM in passive movements may be due to the fact that this score measures the overall stiffness, caused by spasticity and by biomechanical changes in the muscle and muscle shortening. If the non-EMG-dependent stiffness is the main determinant of the stiffness, no correlation would be expected between the EMG-dependent scores on spasticity and the Ashworth tonus score. This finding is in accordance with the results of previous studies [8].

The spasm frequency score showed only a positive correlation with DSR of the gastrocnemius muscle. In most patients spasticity is most pronounced in this muscle. This finding supports the validity of this measurement. Overall the correlation between the spasm score and the EMG-dependent measurements is weak. The absolute value of surface EMG depends mainly on patient characteristics such as the thickness of the subcutaneous fat layer and the mass of the muscles [11, 26]. The intersubject variation on EMG measurements is wide. For this reason the absolute EMG values correspond only weakly with clinical scores of the severity of spasticity. Another explanation for the poor correlation with the clinical spasm score is probably that the spasm score rates the presence of spontaneous spasms, but the pathophysiology of spontaneous spasms differs from that of the raised SRA. This could be another reason for the poor correlation between the scores expressing SRA and spasm scores.

Intrathecal baclofen administration significantly reduced the SRA on all scores comparing the baseline measurements with the clinically optimal dose of baclofen. Moreover, there was a clear dose-dependent response. The scores changed in the expected way, which confirms the construct validity of the RM test. A significant dose-dependent reduction in the scores expressing spasticity and the high values of the power indicate a 
high sensitivity to change on the RM test. The results support the validity of the RM test for objective evaluation of the effect of a bolus dose of intrathecal baclofen on spasticity.

\section{Conclusion}

The RM test is a useful clinical tool to measure objectively the effect of intrathecal baclofen administration on spasticity in patients with an upper motor neuron syndrome.
Acknowledgements Patients were treated by a clinical task group (members Prof. C. Polman, MD PhD, Department of Neurology; S. Egeler, MD, Department of Neurosurgery; Prof. W. Zuurmond, MD PhD, Department of Anesthesiology). The measurements were carried out with the technical support of J. Harlaar, MSc, $\mathrm{PhD}$, Head of the Movement Laboratory.

\section{References}

1. Abel NA, Smith RA (1994) Intrathecal baclofen for treatment of intractable spinal spasticity. Arch Phys Med Rehabil 75:54-58

2. Ashworth B (1964) Preliminary trial of carisoprodol in multiple sclerosis. Practitioner 192:540-542

3. Becher J, Harlaar J, Vogelaar TW (1988) Assessment of muscle function in hemiplegic and healthy subjects by kinesiologic EMG-registration during repetitive movements. In: Wallinga $\mathrm{W}$, Boom HBK, Vries de J (eds) Electrophysiological kinesiology. Excerpta Medica, Amsterdam, pp 357-360

4. Becher JG, Harlaar J, Lankhorst GJ, Vogelaar TW (1998) Measurement of impaired muscle function of the gastrocnemius, soleus, and tibialis anterior muscles in spastic hemiplegia: a preliminary study. J Rehabil Res Dev 35:314-326

5. Campbell SK, Almeida GL, Penn RD, Corcos DM (1995) The effects of intrathecally administered baclofen on function in patients with spasticity. Phys Ther 75:352-362

6. Coffey RJ, Cahill D, Steers W (1993) Intrathecal baclofen for intractable spasticity of spinal origin: results of a long-term multicenter study. J Neurosurg 78:226-232

7. Ditunno JF (1992) Standards for neurological and functional classification of spinal cord injury. American Spinal Injury Association, Chicago
8. Ibrahim IK, Berger W, Trippel M, Dietz V (1993) Stretch-induced electromyographic activity and torque in spastic elbow muscles. Differential modulation of reflex activity in passive and active motor tasks. Brain 116:971-989

9. Katz RT (1988) Management of spasticity. Am J Phys Med Rehabil 67: 108-116

10. Katz RT, Rymer WZ (1989) Spastic hypertonia: mechanisms and measurement. Arch Phys Med Rehabil 70: 144-155

11. Kleissen RFM, Hermens HJ, Exter den T, Kreek de JE (1989) Simultaneous measurement of surface EMG and movements for clinical use. Med Biol Eng Comput 27:291-297

12. Kurtzke JF (1965) Further notes on disability evaluation in multiple sclerosis, with scale modifications. Neurology 15:654-661

13. Lance JW (1980) Symposium synopsis. In: Feldman RG, Young RR, Koella WP (eds) Spasticity: disordered motor control. Year Book Medical, Miami, pp 485-500

14. Lazorthes Y, Sallerin-Caute B, Verdie J (1990) Chronic intrathecal baclofen administration for control of severe spasticity. J Neurosurg 72:393-402

15. Lehmann JF, Price R, Hinderer S (1989) Spasticity: quantitative measurements as a basis for assessing effectiveness of therapeutic intervention. Arch Phys Med Rehabil 70:6-15

16. Loubser PG, Narayan RK, Sandin KJ, Donovan WH, Russell KD (1991) Continuous infusion of intrathecal baclofen: long-term effects on spasticity in spinal cord injury. Paraplegia 29: 48-64

17. Meinders M, Price R, Lehmann JF, Questad KA (1996) The stretch reflex response in the normal and spastic ankle: effect of ankle position. Arch Phys Med Rehabil 77:487-492
18. Meythaler JM, Steers WD, Tuel SM, Cross LL, Haworth CS (1992) Continuous intrathecal baclofen in spinal cord spasticity. A prospective study. Am J Phys Med Rehabil 71:321-327

19. Ochs G, Struppler A, Meyerson BA (1989) Intrathecal baclofen for longterm treatment of spasticity: a multicentre study. J Neurol Neurosurg Psychiatry 52:933-939

20. Penn RD (1992) Intrathecal baclofen for spasticity of spinal origin: seven years of experience. J Neurosurg 77 : 236-240

21. Price R, Lehmann JF, Boswell-Bessette S, Burleigh A, deLateur BJ (1993) Influence of cryotherapy on spasticity at the human ankle. Arch Phys Med Rehabil 74:300-304

22. Sahrmann SA, Norton BJ (1977) The relationship of voluntary movement to spasticity in the upper motor neuron syndrome. Ann Neurol 2:460-465

23. Shiavi R, Green N (1983) Ensemble averaging of locomotor electromyographic patterns using interpolation. Med Biol Eng Comput 21:573-578

24. Stewart-Wynne EG, Silbert PL, Buffery S, Perlman D, Tan E (1991) Intrathecal baclofen for severe spasticity: five years experience. Clin Exp Neurol 28:244-255

25. Streiner DL, Norman GR (1994) Health measurement scales: a practical guide to their development and use. Oxford University Press, Oxford

26. Turker KS (1993) Electromyography: some methodological problems and issues. Phys Ther 73:698-710 